\title{
Diversidade epifítica da flora montana de samambaias e licófitas do Parque Nacional do Itatiaia, Brasil
}

Floristic diversity of epiphitic ferns and lycophytes of montane forest at Itatiaia National Park, Brazil

\author{
Thiago Vieira Costa ${ }^{1,4}$, Elaine Ribeiro Damasceno ${ }^{2}$ \& Lana da Silva Sylvestre ${ }^{2,3}$
}

\begin{abstract}
Resumo
O Parque Nacional do Itatiaia está localizado no sudeste do Brasil, na parte mais elevada da Serra da Mantiqueira. Uma extensa área do Parque é coberta por Floresta Ombrófila Densa Montana, sendo extremamente rica em espécies epifíticas. O objetivo deste trabalho foi reconhecer as espécies de samambaias e licófitas epifíticas com altos índices de densidade e analisar a abundância dessas plantas em três faixas altitudinais. Foram estabelecidos três sítios de amostragem entre 800 e $1.200 \mathrm{~m}$ com intervalos de $200 \mathrm{~m}$. Em cada sítio foram estabelecidas 30 parcelas de $25 \mathrm{~m}^{2}$. Os forófitos foram considerados como unidade amostral natural das plantas inventariadas na base de seus troncos ( $0-2 \mathrm{~m}$ de altura). Trinta e seis espécies de samambaias e licófitas epifíticas distribuídas em 20 gêneros e nove famílias foram encontradas. As famílias com elevada riqueza de espécies foram Aspleniaceae e Polypodiaceae. Campyloneurum lapathifolium, Mickelia scandens, Blechnum acutum e Didymoglossum reptans foram as espécies mais abundantes. A faixa de $800 \mathrm{~m}$ mostrou maior diversidade que as demais.

Palavras-chave: epífitos vasculares, Floresta Atlântica, gradiente altitudinal, pteridoflora.
\end{abstract}

\begin{abstract}
The Itatiaia National Park is located in southeastern Brazil, in the highest part of Mantiqueira Mountain Range. A large area of the Park is covered by a Dense Montane Rain Forest, being extremely rich in epiphytic species. The aim of this work was to recognize the species of ferns and lycophytes with higher density index and analyze the abundance of these plants in three elevation zones. It was established three sampling sites between 800 and $1200 \mathrm{~m}$ at intervals of $200 \mathrm{~m}$. At each site were established 30 plots of $25 \mathrm{~m}^{2}$. Phorophytes were considered natural sample unit of inventoried plants at base of their trunks ( $0-2 \mathrm{~m}$ high). Thirty-six species of epiphytic ferns and lycophytes distributed in 20 genera and nine families were found. The families with highest species richness were Aspleniaceae and Polypodiaceae. Campyloneurum lapathifolium, Mickelia scandens, Blechnum acutum, and Didymoglossum reptans were the most abundant species. The $800 \mathrm{~m}$ zone showed a higher diversity than the others. Key words: vascular epiphytes, Atlantic Forest, elevational gradient, ferns, lycophytes.
\end{abstract}

\section{Introdução}

Epífitos são plantas que passam a maior parte ou todo o tempo de sua vida sobre outras plantas sem obter nutrientes das mesmas (Benzing 1987). Especialmente nas florestas tropicais úmidas, elas compõem parte significativa da flora e sua presença e abundância são frequentemente utilizadas como características de tais florestas (Johansson 1974).
Além de formarem um dos maiores componentes da diversidade nesses ambientes, a comunidade epifitica é a primeira destinada ao declínio quando há ocorrência de perturbações nos ecossistemas florestais (Dubuisson et al. 2009). As espécies de Orchidaceae representam cerca de $73 \%$ do total de epífitos vasculares, enquanto Bromeliaceae e Polypodiaceae geralmente são citadas em segundo e terceiro lugares,

\footnotetext{
${ }^{1}$ Universidade Federal Rural do Rio de Janeiro, Inst. Ciências Biológicas e da Saúde, Depto. Botânica, Rod. BR-465, Km 7, 23897-000, Seropédica, RJ, Brasil.

${ }^{2}$ Universidade Federal do Rio de Janeiro, Museu Nacional, Prog. Pós-graduação em Ciências Biológicas (Botânica), Quinta da Boa Vista, s/n, São Cristóvão, 20940-040, Rio de Janeiro, RJ, Brasil.

${ }^{3}$ Universidade Federal do Rio de Janeiro, Inst. Biologia, Depto. Botânica, CCS Bl. A, Cidade Universitária, 21941-902, Rio de Janeiro, RJ, Brasil.

${ }^{4}$ Autor para correspondência: costatv@live.com
} 
respectivamente, nessa fitofisionomia (Fontoura et al. 1997; Dubuisson et al. 2009). Por outro lado, as samambaias são o segundo grupo de plantas vasculares em termos de riqueza epifítica, com $29 \%$ das espécies ocupando esse hábito. Além disso, associadas às outras plantas vasculares sem sementes, elas podem representar cerca de 36 a $72 \%$ desta riqueza chegando a ser mais ricas que as orquídeas em muitas florestas ombrófilas paleotropicais (Dubuisson et al. 2009). Benzing (1989) ainda considerando as Pteridófitas em sua concepção tradicional, cita que pelo menos nove famílias possuem representantes epifíticos. Entretanto, com base no sistema de classificação de Smith et al. (2006, 2008), podemos afirmar que duas das três famílias de licófitas e dez das 42 de samambaias possuem espécies epifíticas. Polypodiaceae, a família mais derivada dentre as samambaias, é formada predominantemente por essas plantas (Schuettpelz \& Pryer 2009).

Estudos têm mostrado que, ao longo de um gradiente altitudinal, a distribuição da riqueza pode apresentar quatro padrões distintos, a saber: decrescente, baixo platô, baixo platô com pico nas médias elevações e, por fim, o pico em médias elevações (Rahbek 1995; McCain \& Grytnes 2010). Caracterizado por um pico unimodal em altitudes intermediárias (acima de $300 \mathrm{~m}$ ) com $25 \%$ ou mais de espécies do que na base e no topo de uma montanha, esse último padrão é o esperado para os epífitos vasculares, como já demonstrado por Krömer et al. (2005) e Acharya et al. (2011). Além disso, outros grupos de seres vivos evidenciam o mesmo modelo de distribuição de riqueza (Grytnes et al. 2006; Kluge et al. 2006; Grau et al. 2007).

Dusén (1903) foi o primeiro pesquisador a divulgar uma listagem preliminar das samambaias e licófitas ocorrentes no Parque Nacional do Itatiaia. Nesta lista, já era possível notar a importância dos epífitos, que estavam representados por quase $50 \%$ das espécies inventariadas. Brade (1942, 1954, 1956) desenvolveu estudos com abordagem florística, apresentando comentários a respeito da distribuição geográfica dos táxons e suas possíveis rotas de migração. Em um de seus estudos, Brade (1942) afirmou que aproximadamente $21 \%$ das espécies até então conhecidas para a região eram representantes do componente epifítico. Todas as espécies pertenciam à Hymenophyllaceae e Polypodiaceae, embora essa última tenha sido reconhecida com uma circunscrição mais ampla que a adotada atualmente por Smith et al. (2006), com muitas espécies distribuídas basicamente nos gêneros Asplenium, Elaphoglossum e Polypodium (sensu lato). Mais recentemente, Sylvestre (dados não publicados) indicou a presença de 308 espécies de samambaias e licófitas para o
Parque, sendo $49 \%$ epífitos. Outros estudos relevantes foram os de Condack \& Sylvestre (2009) e Ramos \& Sylvestre (2010), que abordaram a questão da estrutura comunitária de samambaias na Floresta Alto-Montana e o tratamento taxonômico das espécies de Lycopodiaceae do Parque, respectivamente.

Embora o conhecimento da flora de samambaias e licófitas no Brasil tenha mostrado um grande avanço nos últimos anos (Prado et al. 2015), estudos mais detalhados são necessários para se entender a dinâmica dessas plantas nas florestas pluviais. Pesquisas nesse sentido, e que enfoquem o epifitismo, se tornam ainda mais prementes, visto que esse é um dos grupos vegetais mais conspícuos nessas formações florestais. Somado a isso está o fato de estarem inseridos na área de maior diversidade brasileira de samambaias e um dos hotspots de biodiversidade do planeta (Myers et al. 2000), a Mata Atlântica.

Portanto, este estudo teve por objetivos realizar um levantamento florístico e análise estrutural buscando reconhecer as espécies de samambaias e licófitas epifíticas com elevados níveis de densidade e avaliar a abundância dessas plantas, em três faixas altitudinais, no sub-bosque de um trecho de Floresta Ombrófila Densa Montana no Parque Nacional do Itatiaia.

\section{Material e Métodos}

Área de estudo

O Parque Nacional do Itatiaia (PNI) é uma Unidade de Conservação de proteção integral e localiza-se na região sudeste do Brasil, na Serra da Mantiqueira, abrangendo o sudoeste do Rio de Janeiro e o sul de Minas Gerais $\left(22^{\circ} 14^{\prime} 33,2^{\prime \prime} \mathrm{N}\right.$ a $22^{\circ} 27^{\prime} 54,1^{\prime \prime} \mathrm{S}$ e $44^{\circ} 34^{\prime} 03,5^{\prime \prime} \mathrm{E}$ a $\left.44^{\circ} 46^{\prime} 10,8^{\prime \prime} \mathrm{W}\right)$. Possui relevo montanhoso e escarpado, entre 540 e 2.791,55 m em relação ao nível do mar, e uma área de 28.084,3 ha (Barreto et al. 2013). Segundo a classificação adotada por Veloso et al. (1991), a Floresta Ombrófila Densa ocorrente no PNI pode ser classificada em Montana, entre 500 e $1.500 \mathrm{~m}$ de altitude, e Alto-Montana, acima de $1.500 \mathrm{~m}$. O presente trabalho foi realizado nos domínios da Floresta Montana. A área de estudo foi dividida em três faixas altimétricas, com diferença de $200 \mathrm{~m}$ entre elas. A primeira foi implantada a $800 \mathrm{~m}$ de altitude, a segunda a $1.000 \mathrm{~m}$ e a terceira a $1.200 \mathrm{~m}$.

\section{Amostragem e análise de dados}

Desenvolvida entre os meses de outubro e dezembro, com até duas expedições de campo por mês, a amostragem foi realizada no sub-bosque da floresta por meio do estabelecimento de parcelas não contíguas de $25 \mathrm{~m}^{2}(5 \mathrm{~m} \times 5 \mathrm{~m})$ com distância média 
de $30 \mathrm{~m}$ entre elas. Em cada faixa altitudinal foram implantadas 30 parcelas, totalizando $750 \mathrm{~m}^{2}$ por faixa e $2.250 \mathrm{~m}^{2}$ em toda a área amostral. Foi registrado o número de indivíduos de cada espécie epifítica localizada até o limite máximo de $2 \mathrm{~m}$ de altura no forófito. Este foi considerado unidade amostral natural dos táxons (Giongo \& Waechter 2004). Plantas encontradas formando pequenas touceiras sobre os forófitos foram consideradas como apenas um indivíduo a fim de padronizar a amostragem. Todos os forófitos que continham epífitos foram incluídos, independente do seu diâmetro. Os forófitos mortos, porém eretos, e os forófitos decumbentes, como as lianas, também foram contabilizados, sendo, neste caso, medida a altura do epífito em relação ao solo. Epífitos ocorrentes em forófitos caídos no chão foram desconsiderados, uma vez que esta amostragem é inadequada para a estimativa da diversidade de comunidades epifíticas (Gradstein et al. 2003).

Foram computados o número de indivíduos epifíticos (Ni) e o número de forófitos onde cada espécie ocorreu (Ui). Os parâmetros fitossociológicos utilizados foram: densidade (abundância) específica relativa $((\mathrm{Ni} / \Sigma \mathrm{Ni}) \times 100)$, frequência absoluta $((\mathrm{Ui} /$ $\Sigma$ Ui $) \times 100)$ e frequência específica relativa ((Fai/ $\Sigma \mathrm{Fa}) \times 100$ ). Para a análise de diversidade foi utilizado o índice de diversidade de Shannon, obtido a partir do programa PAST 3.08 (Hammer et al. 2001). Para a análise de similaridade foi utilizado o coeficiente de dissimilaridade de Bray-Curtis (Bray \& Curtis 1957), também gerado no software citado anteriormente. Para fazer comparações diretas entre as comunidades de epífitos, foi elaborada uma curva de rarefação para cada altitude a partir do estimador não-paramétrico Chao de primeira ordem, utilizando para tal o programa EstimateS 9.1.0 (Colwell 2013). As espécies epifíticas foram classificadas em categorias ecológicas, de acordo com sua relação com o forófito (Benzing 1990), a saber: epífito verdadeiro, epífito facultativo e epífito acidental. As espécies trepadeiras dos gêneros Lomariopsis, Mickelia e Polybotrya, consideradas tradicionalmente como hemiepífitos, foram incluídas na amostragem, devido compartilharem o mesmo extrato que as plantas epifiticas.

As famílias e gêneros aqui reconhecidos seguem o sistema de classificação de Smith et al. $(2006,2008)$. Os táxons foram identificados a partir de bibliografia específica para cada grupo, bem como pela comparação com espécimes depositados em herbários. O material foi coletado e herborizado segundo técnicas descritas para plantas vasculares sem sementes (Silva 1984) e incluído nos acervos dos Herbários do Jardim Botânico do Rio de Janeiro (RB) e do Departamento de Botânica da Universidade Federal Rural do Rio de Janeiro (RBR).

\section{Resultados}

Foram encontradas 34 espécies epifíticas de samambaias e duas de licófitas, estando distribuídas em 20 gêneros e nove famílias. Aspleniaceae e Polypodiaceae foram as que apresentaram maior riqueza específica, cada uma com nove espécies, somando $50 \%$ do total. Dryopteridaceae e Hymenophyllaceae também foram bem representadas, com seis espécies cada. As demais famílias foram representadas por apenas uma ou duas espécies (Tab. 1).

Tabela 1 - Famílias e espécies de samambaias e licófitas epifíticas inventariadas em um trecho de Floresta Ombrófila Densa Montana do Parque Nacional do Itatiaia. Classificação por categorias ecológicas: VER = epífito verdadeiro, $\mathrm{FAC}=$ epífito facultativo, $\mathrm{ACI}=$ epífito acidental e TRE $=$ trepadeira.

Table 1 - Families and species of epiphytic ferns and lycophytes inventoried on a Dense Ombrophilous Forest at Itatiaia National Park. Rating ecological categories: $\mathrm{VER}=$ truly epiphytic, $\mathrm{FAC}=$ facultative epiphytic, $\mathrm{ACI}=$ accidental epiphytic and $\mathrm{TRE}=\mathrm{climbing}$.

\begin{tabular}{|c|c|c|c|c|c|}
\hline \multirow{2}{*}{ Espécies } & \multicolumn{3}{|c|}{ Faixas altitudinais } & \multirow{2}{*}{$\begin{array}{c}\text { Categorias } \\
\text { ecológias }\end{array}$} & \multirow{2}{*}{ Material testemunho } \\
\hline & 800 & 1000 & 1200 & & \\
\hline \multicolumn{6}{|l|}{ Anemiaceae } \\
\hline Anemia phyllitidis (L.) Sw. & & $\mathrm{X}$ & & $\mathrm{ACI}$ & Damasceno \& Costa 187 \\
\hline \multicolumn{6}{|l|}{ Aspleniaceae } \\
\hline Asplenium auriculatum Sw. & $\mathrm{X}$ & $\mathrm{X}$ & & FAC & Damasceno \& Costa 228, 280 \\
\hline Asplenium harpeodes Kunze & & $X$ & & FAC & Damasceno \& Costa 186 \\
\hline Asplenium kunzeanum Klotzsch ex Rosenst & & $X$ & $\mathrm{X}$ & FAC & Damasceno \& Costa 171, 179, 197 \\
\hline Asplenium mucronatum C. Presl & $\mathrm{X}$ & $\mathrm{X}$ & & FAC & Damasceno \& Costa 220 e 238 \\
\hline
\end{tabular}




\begin{tabular}{|c|c|c|c|c|c|}
\hline \multirow{2}{*}{ Espécies } & \multicolumn{3}{|c|}{ Faixas altitudinais } & \multirow{2}{*}{$\begin{array}{l}\text { Categorias } \\
\text { ecológias }\end{array}$} & \multirow{2}{*}{ Material testemunho } \\
\hline & 800 & 1000 & 1200 & & \\
\hline Asplenium oligophyllum Kaulf. & & $\mathrm{X}$ & $\mathrm{X}$ & FAC & Damasceno \& Costa 173, 214 \\
\hline Asplenium pteropus Kaulf. & & & $X$ & VER & Damasceno \& Costa 297 \\
\hline Asplenium raddianum Gaudich. & & & $\mathrm{X}$ & VER & Damasceno \& Costa 290, 296 \\
\hline Asplenium scandicinum Kaulf. & $\mathrm{X}$ & $\mathrm{X}$ & $\mathrm{X}$ & VER & Damasceno \& Costa 144, 199 \\
\hline $\begin{array}{l}\text { Hymenasplenium triquetrum (N. Murak. \& } \\
\text { R.C. Moran) L. Regalado \& Prada }\end{array}$ & & & $\mathrm{X}$ & $\mathrm{ACI}$ & Damasceno \& Costa 133, 183, 288 \\
\hline \multicolumn{6}{|l|}{ Blechnaceae } \\
\hline Blechnum acutum (Desv.) Mett. & $\mathrm{X}$ & $\mathrm{X}$ & $\mathrm{X}$ & FAC & Damasceno \& Costa 167, 226 \\
\hline \multicolumn{6}{|l|}{ Dryopteridaceae } \\
\hline Elaphoglossum glaziovii (Fée) Brade & $\mathrm{X}$ & $\mathrm{X}$ & $\mathrm{X}$ & VER & Damasceno \& Costa 162, 216, 247 \\
\hline Elaphoglossum luridum (Fée) Christ & & $\mathrm{X}$ & & VER & Damasceno \& Costa 191 \\
\hline Mickelia scandens (Raddi) R.C. Moran et al. & $\mathrm{X}$ & $\mathrm{X}$ & & TRE & Damasceno \& Costa 219 \\
\hline Olfersia cervina (L.) Kunze & & & $\mathrm{X}$ & $\mathrm{ACI}$ & Damasceno \& Costa 154, 158 \\
\hline Polybotrya cylindrica Kaulf. & $\mathrm{X}$ & & & TRE & Damasceno \& Costa 237 \\
\hline Polybotrya speciosa Schott & & $\mathrm{X}$ & $\mathrm{X}$ & TRE & Damasceno \& Costa 165, 189 \\
\hline \multicolumn{6}{|l|}{ Hymenophyllaceae } \\
\hline Didymoglossum reptans (Sw.) C. Presl & $\mathrm{X}$ & $\mathrm{X}$ & $\mathrm{X}$ & FAC & Damasceno \& Costa 148, 190, 294 \\
\hline Hymenophyllum hirsutum (L.) Sw. & $\mathrm{X}$ & & & VER & Damasceno \& Costa 246 \\
\hline $\begin{array}{l}\text { Polyphlebium angustatum (Carmich.) } \\
\text { Ebihara \& Dubuisson }\end{array}$ & $\mathrm{X}$ & $X$ & $\mathrm{X}$ & VER & Damasceno \& Costa 151, 193, 265 \\
\hline $\begin{array}{l}\text { Polyphlebium pyxidiferum (L.) Ebihara \& } \\
\text { Dubuisson }\end{array}$ & $\mathrm{X}$ & $X$ & $\mathrm{X}$ & FAC & Damasceno \& Costa 196, 272, 291 \\
\hline Trichomanes polypodioides Raddi & & $\mathrm{X}$ & & VER & Damasceno \& Costa 233 \\
\hline Vandenboschia radicans (Sw.) Copel. & $\mathrm{X}$ & $\mathrm{X}$ & $\mathrm{X}$ & FAC & Damasceno \& Costa 143, 204, 212, 293 \\
\hline \multicolumn{6}{|l|}{ Lomariopsidaceae } \\
\hline Lomariopsis marginata (Schrad.) Kuhn & $\mathrm{X}$ & & & TRE & Damasceno \& Costa 147 \\
\hline \multicolumn{6}{|l|}{ Polypodiaceae } \\
\hline Campyloneurum lapathifolium (Poir.) Ching & $\mathrm{X}$ & $X$ & $\mathrm{X}$ & FAC & Damasceno \& Costa 138, 161, 185, 195 \\
\hline Campyloneurum nitidum (Kaulf.) C. Presl & $\mathrm{X}$ & $\mathrm{X}$ & $\mathrm{X}$ & FAC & Damasceno \& Costa 224, 242, 287 \\
\hline Pecluma recurvata (Kaulf.) M.G. Price & $\mathrm{X}$ & & & VER & Damasceno \& Costa 285 \\
\hline Pecluma sicca (Lindm.) M.G. Price & & $X$ & & VER & Damasceno \& Costa 188 \\
\hline Pecluma truncorum (Lindm.) M.G. Price & $\mathrm{X}$ & $\mathrm{X}$ & $\mathrm{X}$ & VER & Damasceno \& Costa 213 \\
\hline Pleopeltis astrolepis (Liebm.) Fourn. & & $\mathrm{X}$ & & VER & Damasceno \& Costa 225 \\
\hline $\begin{array}{l}\text { Pleopeltis macrocarpa (Bory ex Willd.) } \\
\text { Kaulf. }\end{array}$ & & & $\mathrm{X}$ & FAC & Damasceno \& Costa 174 \\
\hline $\begin{array}{l}\text { Serpocaulon catharinae (Langsd. \& Fisch.) } \\
\text { A.R.Sm. }\end{array}$ & & & $\mathrm{X}$ & FAC & Damasceno \& Costa 172 \\
\hline $\begin{array}{l}\text { Serpocaulon fraxinifolium (Jacq.) A.R.Sm. } \\
\text { Pteridaceae }\end{array}$ & $\mathrm{X}$ & $\mathrm{X}$ & & VER & Damasceno \& Costa 198, 279 \\
\hline Polytaenium cajenense (Desv.) Benedict & $\mathrm{X}$ & & & VER & Damasceno \& Costa 145 \\
\hline Selaginellaceae & & & & & \\
\hline Selaginella contigua Baker & & & $\mathrm{X}$ & $\mathrm{ACI}$ & Damasceno \& Costa 178 \\
\hline Selaginella flexuosa Spring & $\mathrm{X}$ & & & $\mathrm{ACI}$ & Damasceno \& Costa 245 \\
\hline
\end{tabular}


Analisando as três faixas altitudinais em conjunto (Tab. 2), Campyloneurum lapathifolium foi a samambaia com maior valor de abundância. Entretanto, quando se considera este parâmetro por faixa altitudinal (Fig. 1), nota-se que na altitude de $800 \mathrm{~m}$ ela é substituída por Mickelia scandens. Polypodiaceae apresentou elevados índices, não somente quantitativos (abundância) como qualitativos (riqueza) (faixas de 1.000 e 1.200 $\mathrm{m})$. Dryopteridaceae se destacou na faixa de $800 \mathrm{~m}$ e Aspleniaceae mostrou distribuição aparentemente uniforme no decorrer das três faixas altitudinais, com um leve incremento também na faixa de $800 \mathrm{~m}$. Por outro lado, Pteridaceae, Anemiaceae e Selaginellaceae revelaram baixos índices de densidade. Algumas espécies ocorreram nas três faixas altitudinais. Essas plantas representaram $27,78 \%$ do total das espécies inventariadas. Outras, porém, foram exclusivas de cada uma das faixas. Em relação às categorias ecológicas, os epífitos verdadeiros foram os mais representativos $(38,8 \%)$ em toda a área amostral, seguido pelos epífitos facultativos $(36,1 \%)$.

Tabela 2 - Parâmetros fitossociológicos analisados para as samambaias e licófitas epifíticas ocorrentes em um trecho de Floresta Ombrófila Densa Montana do Parque Nacional do Itatiaia, organizados em ordem decrescente de valor de densidade relativa. $\mathrm{Ni}$ = número de indivíduos; $\mathrm{Ui}$ = número de forófitos em que a espécie epifítica ocorreu; $\mathrm{DRi}(\%)$ $=$ densidade relativa; $\mathrm{FAi}(\%)=$ frequência absoluta e $\mathrm{FRi}(\%)=$ frequência relativa.

Table 2 - Phytosociological parameters analyzed to epiphytic ferns and lycophytes occurrent on a Dense Ombrophilous Forest at Itatiaia National Park organized in descending order of relative density. $\mathrm{Ni}=$ number of individuals; Ui = number of phorophytes where the species occurred; DRi $(\%)=$ relative density; FAi $(\%)=$ absolute frequency and $\mathrm{FRi}(\%)=$ relative frequency.

\begin{tabular}{lccccc}
\hline Espécie & Ni & Ui & DRi(\%) & FAi(\%) & FRi(\%) \\
\hline Campyloneurum lapathifolium (Poir.) Ching & 245 & 183 & 33,52 & 46,45 & 30,45 \\
Mickelia scandens (Raddi) R.C. Moran et al. & 64 & 61 & 8,76 & 15,48 & 10,15 \\
Didymoglossum reptans (Sw.) C. Presl & 56 & 48 & 7,66 & 12,18 & 7,99 \\
Blechnum acutum (Desv.) Mett. & 55 & 47 & 7,52 & 11,93 & 7,82 \\
Polyphlebium angustatum (Carmich.) Ebihara \& Dubuisson & 32 & 32 & 4,38 & 8,12 & 5,32 \\
Campyloneurum nitidum (Kaulf.) C. Presl & 30 & 27 & 4,10 & 6,85 & 4,49 \\
Asplenium kunzeanum Klotzsch ex Rosenst & 30 & 23 & 4,10 & 5,84 & 3,83 \\
Serpocaulon fraxinifolium (Jacq.) A.R.Sm. & 22 & 20 & 3,01 & 5,08 & 3,33 \\
Vandenboschia radicans (Sw.) Copel. & 22 & 20 & 3,01 & 5,08 & 3,33 \\
Asplenium mucronatum C. Presl & 21 & 17 & 2,87 & 4,31 & 2,83 \\
Polybotrya speciosa Schott & 18 & 16 & 2,46 & 4,06 & 2,66 \\
Lomariopsis marginata (Schrad.) Kuhn & 15 & 13 & 2,05 & 3,30 & 2,16 \\
Asplenium scandicinum Kaulf. & 15 & 12 & 2,05 & 3,05 & 2,00 \\
Asplenium auriculatum Sw. & 15 & 8 & 2,05 & 2,03 & 1,33 \\
Polyphlebium pyxidiferum (L.) Ebihara \& Dubuisson & 12 & 12 & 1,64 & 3,05 & 2,00 \\
Pecluma truncorum (Lindm.) M.G. Price & 11 & 9 & 1,50 & 2,28 & 1,50 \\
Polybotrya cylindrica Kaulf. & 8 & 7 & 1,09 & 1,78 & 1,16 \\
Polytaenium cajenense (Desv.) Benedict & 8 & 5 & 1,09 & 1,27 & 0,83 \\
Asplenium oligophyllum Kaulf. & 8 & 4 & 1,09 & 1,02 & 0,67 \\
Asplenium harpeodes Kunze & 7 & 7 & 0,96 & 1,78 & 1,16 \\
Pecluma sicca (Lindm.) M.G. Price & 7 & 3 & 0,96 & 0,76 & 0,50 \\
Elaphoglossum glaziovii (Fée) Brade & 5 & 4 & 0,68 & 1,02 & 0,67 \\
Asplenium raddianum Gaudich. & 4 & 4 & 0,55 & 1,02 & 0,67 \\
Pleopeltis astrolepis (Liebm.) Fourn. & 4 & 3 & 0,55 & 0,76 & 0,50 \\
Serpocaulon catharinae (Langsd. \& Fisch.) A.R.Sm. & 4 & 3 & 0,55 & 0,76 & 0,50 \\
\hline & & & & \\
& & & &
\end{tabular}




\begin{tabular}{lccccc}
\hline Espécie & Ni & Ui & DRi(\%) & FAi(\%) & FRi(\%) \\
\hline Olfersia cervina (L.) Kunze & 2 & 2 & 0,27 & 0,51 & 0,33 \\
Selaginella flexuosa Spring & 2 & 2 & 0,27 & 0,51 & 0,33 \\
Anemia phyllitidis (L.) Sw. & 1 & 1 & 0,14 & 0,25 & 0,17 \\
Asplenium pteropus Kaulf. & 1 & 1 & 0,14 & 0,25 & 0,17 \\
Elaphoglossum luridum (Fée) Christ & 1 & 1 & 0,14 & 0,25 & 0,17 \\
Hymenasplenium triquetrum (N. Murak. \& R.C. Moran) L. Regalado \& Prada & 1 & 1 & 0,14 & 0,25 & 0,17 \\
Hymenophyllum hirsutum (L.) Sw. & 1 & 1 & 0,14 & 0,25 & 0,17 \\
Pecluma recurvata (Kaulf.) M.G. Price & 1 & 1 & 0,14 & 0,25 & 0,17 \\
Pleopeltis macrocarpa (Bory ex Willd.) Kaulf. & 1 & 1 & 0,14 & 0,25 & 0,17 \\
Selaginella contigua Baker & 1 & 1 & 0,14 & 0,25 & 0,17 \\
Trichomanes polypodioides Raddi & 1 & 1 & 0,14 & 0,25 & 0,17 \\
Total & $\mathbf{7 3 1}$ & $\mathbf{3 9 4}$ & $\mathbf{1 0 0}$ & $\mathbf{1 5 2 , 5 4}$ & $\mathbf{1 0 0}$ \\
\hline
\end{tabular}

Em relação às curvas de rarefação, as faixas altitudinais evidenciaram diferenças marcantes. (Fig. 2). A única com clara tendência à assíntota horizontal foi a de $800 \mathrm{~m}$. Em $1.000 \mathrm{~m}$, embora a inclinação não tenha sido acentuada, a curva não revelou tendência à estabilização, apresentando certa sinuosidade. Por

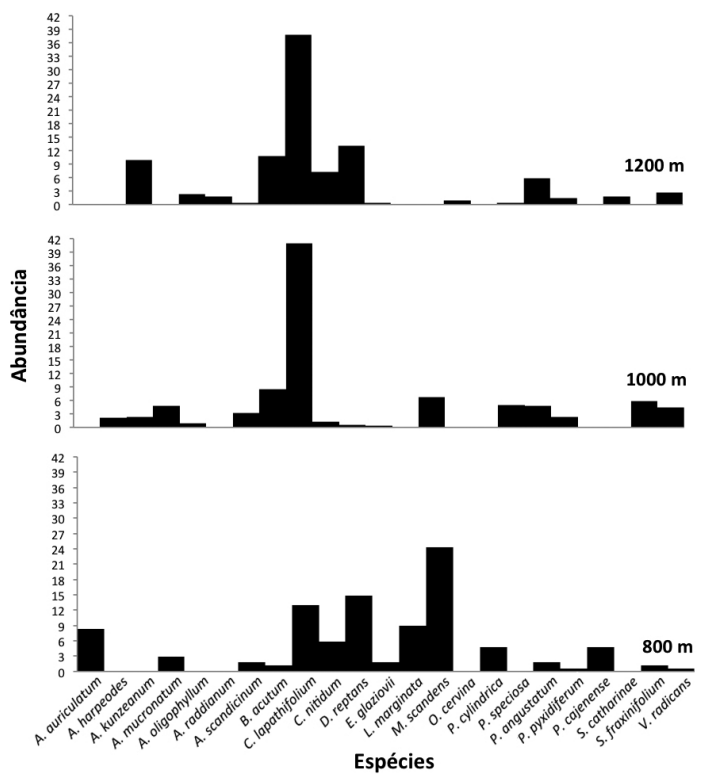

Figura 1 - Densidade relativa (abundância) das samambaias e licófitas epifíticas ocorrentes em um trecho de Floresta Ombrófila Densa Montana do Parque Nacional do Itatiaia, organizada por faixa altitudinal. Figure 1 - Relative density (abundance) of epiphytic ferns and lycophytes occurrent on a Dense Ombrophilous Forest at Itatiaia National Park, organized by altitudinal zones. último, a altitude de $1.200 \mathrm{~m}$ apresentou a maior inclinação dentre as rarefações.

Oíndice de diversidade de Shannon demonstrou que a faixa de $800 \mathrm{~m}$ possui uma pteridoflora epifítica mais diversa $\left(\mathrm{H}^{\prime}=2,419\right)$. $\mathrm{O}$ mesmo índice foi levemente inferior para as áreas de 1.000 e $1.200 \mathrm{~m}\left(\mathrm{H}^{\prime}\right.$ $=2,28$ e 2,116, respectivamente). Com alta correlação cofenética $(0,9712)$, a análise de similaridade florística evidenciou uma união melhor sustentada entre as duas últimas faixas altimétricas, que obtiveram o maior valor de similaridade entre si (54\%). Ao contrário, o menor valor foi indicado para as duas primeiras altitudes (28\%). Entre as faixas de 800 e $1.200 \mathrm{~m}$ constatou-se similaridade intermediária (35\%).

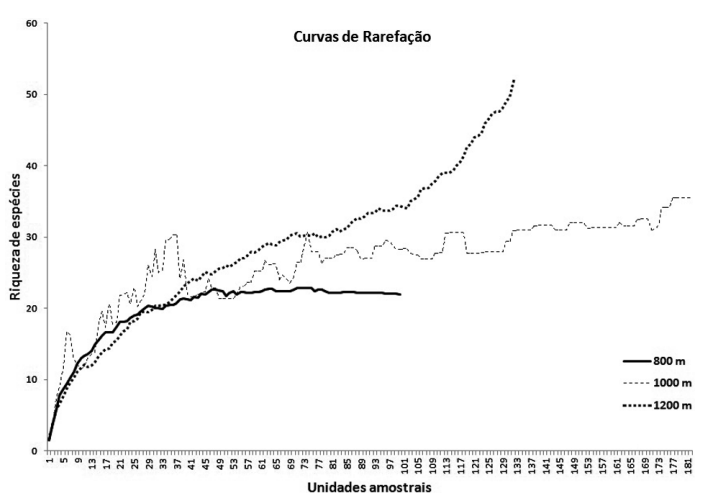

Figura 2 - Comparação entre as curvas de rarefação das três faixas altitudinais amostradas em um trecho de Floresta Ombrófila Densa Montana do Parque Nacional do Itatiaia. Figure 2 - Comparison between rarefaction curves of three sampled elevation zones on a Dense Ombrophilous Forest at Itatiaia National Park. 


\section{Discussão}

Alguns trabalhos apontam Polypodiaceae e Aspleniaceae como famílias mais representativas em levantamentos florísticos de florestas tropicais (Xavier \& Barros 2003; Santiago et al. 2004). Como já esperado, nossos dados convergiram para um cenário semelhante, destacando essas famílias das demais em termos de riqueza. Este fato pode ser explicado pela habilidade que esses táxons têm de se alastrar a partir de gemas que possuem em suas raízes (Moran 2009). Por outro lado, Dryopteridaceae e Hymenophyllaceae são sugeridas como grupos também muito diversos em regiões de Floresta Ombrófila Densa Montana e Alto-Montana (Watkins et al. 2006). Nossos dados corroboram isto, visto que aproximadamente $33 \%$ das espécies estão inseridas nessas duas últimas famílias.

O gênero Campyloneurum é amplamente distribuído em florestas úmidas, podendo ocorrer sobre os mais variados tipos de substrato como, por exemplo, troncos, galhos que pendem sobre riachos, rochas e solos úmidos. Além disso, consegue colonizar áreas em clareiras de florestas degradadas e alcançam grande variação altitudinal, sendo mais comuns acima dos $1.000 \mathrm{~m}$ (Tryon \& Tryon 1982). Essa grande plasticidade sustenta a elevada abundância de $C$. lapathifolium encontrada na área de estudo, pois as características da vegetação em muito se assemelharam ao supracitado. Por outro lado, a samambaia Mickelia scandens, também típica de florestas úmidas e endêmica do Sul e Sudeste do Brasil, é apontada para altitudes que variam entre $0-800 \mathrm{~m}$ (Moran et al. 2010). Nossos dados mostraram um padrão diferente, pois a mesma ocorreu em elevações superiores ao intervalo descrito para a espécie. De modo geral, as características da vegetação nas três faixas não variaram muito, o que pode ter contribuído para o sucesso no estabelecimento de $M$. scandens em altitudes mais elevadas.

Muitos estudos sobre epifitismo têm demonstrado os epífitos verdadeiros como os mais comuns em variadas formações florestais (Dittrich et al. 1999; Kersten \& Silva 2001), assim como em Floresta Ombrófila Densa Montana (Fontoura et al. 1997). O mesmo padrão foi encontrado para as espécies deste estudo. A classificação das plantas nessas categorias ecológicas pode variar de acordo com o autor ou as condições ambientais predominantes no local (Schmitt \& Windisch 2010). Vale ressaltar que esta análise foi realizada com base na ocorrência e distribuição dessas espécies na área de estudo. Desta forma, algumas espécies aqui relacionadas como epífitos verdadeiros podem ser encontradas colonizando outros tipos de substratos em outras áreas de ocorrência de floresta atlântica. Das 14 espécies de epífitos verdadeiros inventariados, oito foram considerados epífitos facultativos em levantamentos realizados em outras localidades. Pecluma recurvata, por exemplo, crescia como epífito e sobre rochas tanto em Serra Bonita, na floresta atlântica sul baiana (Matos et al. 2010), quanto no interior da floresta com araucária e nas formações areníticas do Parque Estadual de Vila Velha, no Paraná (Schwartsburd \& Labiak 2007). Além disso, outras espécies como Asplenium pteropus, Polytaenium cajenense e Serpocaulon fraxinifolium ocorrem como epífitos facultativos em várias localidades ao longo da sua distribuição geográfica (Mickel \& Smith 2004). Algumas espécies que crescem predominantemente sobre o solo podem ser ocasionalmente encontradas como epífitos (acidentais) como, por exemplo, Anemia phyllitidis e Olfersia cervina. Estas espécies conseguem se estabelecer nos forófitos em função condições ambientais apropriadas, tais como alta umidade relativa e disponibilidade de sedimentos acumulados na base dos troncos. A respeito das espécies trepadeiras, estudos têm demonstrado que o termo 'hemiepifitismo' tem sido mal aplicado em samambaias e licófitas, tornando-se confuso muitas vezes, porque para distinção entre os hemiepífitos e as trepadeiras ou epífitas do baixo tronco, é necessária a observação de todos os estágios de vida da planta (Testo \& Sundue 2014). Dessa forma, a maioria das samambaias que tem sido descritas como hemiepifíticas são, na verdade, trepadeiras que nunca perderam a ligação com solo (Canestraro et al. 2014). A região neotropical constitui um dos grandes centros de diversidade de samambaias e licófitas do planeta, pois está associada à elevada riqueza e abundância de espécies (Moran 2008). Embora alguns táxons da nossa amostragem ultrapassem os limites desse domínio, a maior parte dos epífitos encontrados são restritos a essa região biogeográfica. No Brasil, a Mata Atlântica continua sendo o bioma mais rico quando se trata desse grupo de plantas, além de possuir também o maior registro de endemismo (Prado et al. 2015). Esse fato pôde ser observado nos nossos dados, visto que um terço das plantas inventariadas é endêmico do Brasil e estão circunscritas a este domínio, evidenciando a importância que essa área tem para o conhecimento da flora global de samambaias. É importante salientar que as duas espécies verificadas como as mais abundantes e 
que caracterizaram a pteridoflora epifítica fazem parte deste grupo, reforçando a necessidade de conservação dessas florestas.

Em regiões de elevada diversidade, como nas florestas tropicais úmidas, quanto mais indivíduos forem amostrados, maior será a riqueza encontrada, dificultando o estabelecimento de um limite claro para o esforço amostral (Kersten \& Galvão 2011). O mesmo podemos concluir ao observar nossos resultados, pois, com exceção da primeira faixa altitudinal, as curvas de rarefação não revelaram tendência à estabilização. Especialmente em 1.200 $\mathrm{m}$, a acentuada inclinação da curva sugere esta área como a mais promissora a conter os mais elevados índices de riqueza. $\mathrm{O}$ contrário mostrouse evidente na primeira elevação, onde o esforço amostral pode ser considerado satisfatório. A assíntota alcançada em $800 \mathrm{~m}$ propõe variações insignificantes na riqueza esperada para essa área. Provavelmente por estar entre essas duas faixas, a cota de $1.000 \mathrm{~m}$ apresentou o padrão levemente inclinado na curva, indicando que, apesar de grande abundância de epífitos, a riqueza esperada para essa região não seria muito maior que a encontrada no levantamento florístico-estrutural.

A tendência geral para os epífitos é estarem melhor representados nas florestas nebulares de médias elevações e, embora sejam muito abundantes nessas regiões altimétricas, sua densidade não está intimamente relacionada à sua diversidade (Gentry \& Dodson 1987). Nossos resultados apontam nesse sentido, pois as áreas que se destacaram quantitativamente (número de indivíduos em 1.000 e 1.200 m) obtiveram os menores índices de diversidade. $\mathrm{O}$ número de táxons pouco variou nas três faixas de altitude. No entanto, nas duas últimas, foi notória a dominância de poucas espécies, entre elas, Campyloneurum lapathifolium e Blechnum acutum. Em 800 m, por outro lado, embora Mickelia scandens tenha se destacado, a maior uniformidade entre as abundâncias sustenta a maior heterogeneidade desta área e, consequentemente, o valor de Shannon alcançado. Além disso, não obstante a pouca variação da riqueza epifítica nas três faixas, nossos resultados convergem para o padrão de distribuição unimodal-parabólico ou pico em médias elevações. Mesmo com o levantamento florístico de apenas três áreas no gradiente altitudinal, esse modelo pôde ser observado.

Alguns autores consideram altos valores de similaridade florística acima de 30\% (Borgo \& Silva 2003). Porém, para serem consideradas similares em termos de composição, duas áreas necessitam de, pelo menos, 25\% de espécies compartilhadas (Mueller-Dombois \& Ellenberg apud Kunz et al. 2009). Além disso, Salazar et al. (2015) citam que comunidades locais de samambaias à escala regional mostram alto grau de similaridade nas florestas montanas neotropicais. A partir de nossos dados, à luz desses pressupostos, podemos concluir que as floras epifíticas de samambaias e licófitas das três altitudes são significativamente similares. Setenta por cento da riqueza de $800 \mathrm{~m}$ ocorreu em $1.000 \mathrm{~m}$. Esta faixa, por sua vez, conteve $65 \%$ das espécies de $1.200 \mathrm{~m}$. Por fim, 800 e 1.200 m compartilham $50 \%$ de seus epífitos. Esses altos valores de compartilhamento, associados ao baixo número de plantas exclusivas em cada faixa (seis, seis e sete, respectivamente), reforçam a semelhança fitofisionômica destas áreas. Ainda podemos ressaltar que a pouca variação climática e o bom estado de preservação observados no trecho de floresta estudado apontam para estes resultados. É sabido que o papel do espaço e do ambiente sobre a composição de espécies varia de acordo com os diferentes grupos de epífitos (Leitman et al. 2015). No caso das samambaias e licófitas, o ambiente tem mostrado significativamente seus efeitos (Gasper et al. 2015).

Nossos resultados destacam a importância de alguns grupos taxonômicos de samambaias na flora da Mata Atlântica, mostrando que as semelhanças fisionômicas da vegetação pode contribuir para a ocorrência em maior amplitude altitudinal, como ocorreu com Mickelia scandens. Além disso, os epífitos mais plásticos adaptativamente podem colonizar de maneira mais profusa, visto que florestas tropicais apresentam grande diversidade de habitats, tornando essas áreas propensas à homogeneidade taxonômica em alguns casos.

O marco para a maior diversidade de samambaias e licófitas epifíticas está na presença de espécies raras ou pouco abundantes. Portanto, inventários futuros são sugeridos como forma de aumentar o conhecimento a respeito dessas plantas, principalmente com relação a sua ocorrência e distribuição em formações como a Floresta Ombrófila Densa da costa leste sulamericana.

\section{Agradecimentos}

À Fundação Carlos Chagas Filho de Apoio à Pesquisa do Estado do Rio de Janeiro (FAPERJ), os recursos concedidos através do processo E-26/110.054/2008. À Coordenação de Aperfeiçoamento de Pessoal de Nível Superior 
(CAPES), a bolsa de Mestrado concedida à segunda autora. E ao Conselho Nacional de Desenvolvimento Científico e Tecnológico $(\mathrm{CNPq})$, a bolsa de pesquisa concedida ao terceiro autor (Processo 309415/2008-0).

\section{Referências}

Acharya, K.P.; Vetaas, O.R. \& Birks, H.J.B. 2011. Orchid species richness along Himalayan elevational gradients. Journal of Biogeography 38: 1821-1833.

Barreto, C.G.; Campos, J.B.; Roberto, D.M.; Roberto, D.M.; Schwarzstein, N.T.; Alves, G.S.G. \& Coelho, W. 2013. Plano de Manejo do Parque Nacional do Itatiaia. Instituto Chico Mendes de Conservação da Biodiversidade, Brasília. 491p.

Benzing, D.H. 1987. Vascular epiphytism: taxonomic participation and adaptive diversity. Annals of the Missouri Botanical Garden 74: 183-204.

Benzing, D.H. 1989. Vascular Epiphytism in America. In: Lieth, H. \& Werger, M.J.A. (eds.). Tropical Rain Forest Ecosystems. Elsevier Science Publishers B.V., Amsterdan. Pp. 133-154.

Benzing, D.H. 1990. Vascular epiphytes. General biology and related biota. Cambridge University Press, Cambridge. $354 \mathrm{p}$.

Borgo, M \& Silva, S.M. 2003. Epífitos vasculares em fragmentos de Floresta Ombrófila Mista, Curitiba, Paraná, Brasil. Revista Brasileira de Botânica 26: 391-401.

Brade, A.C. 1942. A composição da flora pteridófita do Itatiaia. Contribuição à fitogeografia dessa região. Rodriguésia 6: 29-43.

Brade, A.C. 1954. Contribuição para o conhecimento da flora da Serra do Itatiaia. Arquivos do Jardim Botânico do Rio de Janeiro 13: 63-68.

Brade, A.C. 1956. A Flora do Parque Nacional do Itatiaia. Boletim do Parque Nacional do Itatiaia 5: 1-85.

Bray, J.R. \& Curtis, J.T. 1957. An ordination of the upland forest communities of Southern Wisconsin. Ecological Monographs 27: 325-349.

Canestraro, B.K.; Moran, R.C. \& Watkins Jr, J.E.2014. Reproductive and physiological ecology of climbing and terrestrial Polybotrya (Dryopteridaceae) at the la Selva Biological Station, Costa Rica. International Journal of Plant Sciences 175: 432-441.

Colwell, R.K. 2013. EstimateS: Statistical estimation of species richness and shared species from samples. Version 9. Disponível em <http://purl.oclc.org/ estimates $>$. Acesso em 25 julho 2014.

Condack, J.P.S. \& Sylvestre, L.S. 2009. Structure of fern community in the high mountain forest of Itatiaia National Park, Brazil. In: Verma, S.C.; Khullar, S.P. \& Cheema, H.K. Perspectives in pteridophytes. Bishen Singh Mahendra Pal Singh, Lucknow. Pp. 113-126.
Dittrich, V.A.O.; Kozera, C. \& Menezes-Silva, S. 1999. Levantamento florístico dos epífitos vasculares do Parque Barigüi, Curitiba, Paraná, Brasil. Iheringia, Série Botânica 52: 11-21.

Dubuisson, J.-Y.; Schneider, H. \& Hennequin, S. 2009. Epiphytism in ferns: diversity and history. C.R. Biologies 332: 120-128.

Dusén, P.K.H. 1903. Sur la flore de la Serra do Itatiaya. Arquivos do Museu Nacional 13: 1-119.

Fontoura, T.; Sylvestre, L.S.; Vaz, A.M.S.F. \& Vieira, C.M. 1997. Epífitas vasculares, hemiepífitas e hemiparasitas da Reserva Ecológica de Macaé de Cima. In: Lima, H.C. \& Guedes-Bruni, R.R. (eds.). Serra de Macaé de Cima: Diversidade florística e conservação em Mata Atlântica. Jardim Botânico do Rio de Janeiro, Rio de Janeiro. Pp. 89-101.

Gasper, A.L.; Eisenlohr, P.V. \& Salino, A. 2015. Climaterelated variables and geographic distance affect fern species composition across a vegetation gradient in a shrinking hotspot. Plant Ecology \& Diversity 8: 25-35.

Gentry, A.H. \& Dodson, C.H. 1987. Diversity and biogeography of neotropical vascular epiphytes. Annals of the Missouri Botanical Gardens 74: 205-233.

Giongo, C. \& Waechter, J.L. 2004. Composição florística e estrutura comunitária de epífitos vasculares em uma floresta de galeria na Depressão Central do Rio Grande do Sul. Revista Brasileira de Botânica 27: 563-572.

Gradstein, S.R.; Nadkarni, N.M.; Krömer, T.; Holz, I. \& Nöske, N. 2003. A protocol for rapid and representative sampling of vascular and nonvascular epiphyte diversity of tropical rain forests. Selbyana 24: 105-111.

Grau, O.; Grytnes, J.A. \& Birks, H.J.B. 2007. A comparison of altitudinal species richness patterns of bryophytes with other plant groups in Nepal, Central Himalaya. Journal of Biogeography 34: 1907-1915.

Grytnes, J.A.; Heegaard, E. \& Ihlen, P.G. 2006. Species richness of vascular plants, bryophytes, and lichens along an altitudinal gradient in western Norway. Acta Oecologica 29: 241-246.

Hammer, Ø.; Harper, D.A.T. \& Ryan, P.D. 2001. PAST: Paleontological statistics software package for education and data analysis. Palaeontologia Electronica 4: 9p.

Johansson, D. 1974. Ecology of vascular epiphytes in West African rain forest. Acta Phytogeographica Suecica 59: 1-131.

Kersten, R.A. \& Galvão, F. 2013. Suficiência amostral em inventários florísticos e fitossociológicos. In: Felfili, J.M.; Eisenlohr, P.V.; Melo, M.M.R.F.; Andrade, L.A. \& Meira Neto, J.A.A. (eds.). Fitossociologia no Brasil. Métodos e estudos de casos. Vol. 1. Ed. UFV, Viçosa. Pp. 156-173. 
Kersten, R.A. \& Silva, S.M. 2001. Composição florística e estrutura do componente epifítico vascular em floresta da planície litorânea na Ilha do Mel, Paraná, Brasil. Revista Brasileira de Botânica 24: 213-226.

Kluge, J.; Kessler, M. \& Dunn, R.R. 2006. What drives elevational patterns of diversity? A test of geometric constraints, climate and species pool effects for pteridophytes on an elevational gradient in Costa Rica. Global Ecology and Biogeography 15: 358-371.

Krömer, T.; Kessler, K.; Gradstein, S.R. \& Acebey, A. 2005. Diversity patterns of vascular epiphytes along an elevational gradient in the Andes. Journal of Biogeography 32: 1799-1809.

Kunz, S.H.; Ivanauskas, N.M.; Martins, S.V.; Silva, E. \& Stefanello, D. 2009. Análise da similaridade florística entre florestas do Alto Xingu, da bacia Amazônica e do Planalto Central. Revista Brasileira de Botânica 32: 725-736.

Leitman, P.; Amorim, A.M.; Sansevero, J.B.B. \& Forzza, R.C. 2015. Floristic patterns of epiphytes in the Brazilian Atlantic Forest, a biodiversity hotspot. Botanical Journal of the Linnean Society 179: 587-601.

Matos, F.B.; Amorim, A.M. \& Labiak, P.H. 2010. The ferns and lycophytes of a Montane Tropical Forest in Southern Bahia, Brazil. Journal of the Botanical Research Institute of Texas 4: 333-346.

McCain, C.M. \& Grytnes, J.A. 2010. Elevational gradients in species richness. In: Encyclopedia of Life Sciences (ELS). John Wiley \& Sons, Chichester. 10p.

Mickel, J.T. \& Smith, A.R. 2004. The pteridophytes of Mexico. The New York Botanical Garden Press, New York. 1055p.

Moran, R.C. 2008. Diversity, biogeography, and floristics. In: Ranker, T.A. \& Haufler, C.H. (eds.). Biology and evolution of ferns and lycophytes. Cambridge University Press, New York. Pp. 367-394.

Moran, R.C. 2009. A natural history of ferns. Timber Press, Portland. 302p.

Moran, R.C. \& Smith, A.R. 2001. Phytogeographic relationships between neotropical and AfricanMadagascan pteridophytes. Brittonia 53: 304351.

Moran, R.C.; Labiak, P.H. \& Sundue, M. 2010. Synopsis of Mickelia, a newly recognized genus of bolbitidoid ferns (Dryopteridaceae). Brittonia 62: 337-356.

Myers, N.; Mittermeier, R.A.; Mittermeier, C.G.; Fonseca, G.A.B. \& Kent, J. 2000. Biodiversity hotspots for conservation priorities. Nature 403: 853-858.
Parris, B.S. 2001. Circum-Antarctic continental distribution patterns in pteridophyte species. Brittonia 53: 270-283.

Prado, J.; Sylvestre, L.S.; Labiak, P.H.; Windisch, P.G.; Salino, A.; Barros, I.C.L.; Hirai, R.Y.; Almeida, T.E.; Santiago, A.C.P.; Kieling-Rubio, M.A.; Pereira, A.F.N.; Øllgaard, B.; Ramos, C.G.V.; Mickel, J.T.; Dittrich, V.A.O.; Mynssen, C.M.; Schwartsburd, P.B.; Condack, J.P.S.; Pereira, J.B.S. \& Matos, F.B. 2015. Diversity of ferns and lycophytes in Brazil. Rodriguésia 66: 1073-1083.

Rahbek, C. 1995. The elevational gradient of species richness: a uniform pattern? Ecography 18: 200-205.

Ramos, C.G.V. \& Sylvestre, L.S. 2010. Lycopodiaceae no Parque Nacional do Itatiaia, Rio de Janeiro, Brasil. Acta Botanica Brasilica 24: 25-46.

Rothfels, C.J.; Sundue, M.A.; Kuo, L.; Larsson, A.; Kato, M.; Schuettpelz, E. \& Pryer, K.M. 2012. A revised family-level classification for eupolypod II ferns (Polypodiidae: Polypodiales). Taxon 61: 515-533.

Salazar, L.; Homeier, J.; Kessler, M.; Abrahamczyk, S.; Lehnert, M.; Krömer, T. \& Kluge, J. 2015. Diversity patterns of ferns along elevational gradients in Andean tropical forests. Plant Ecology \& Diversity 8: 13-24.

Santiago, A.C.P.; Barros, I.C.L. \& Sylvestre, L.S. 2004. Pteridófitas ocorrentes em três fragmentos florestais de um brejo de altitude (Bonito, Pernambuco, Brasil). Acta Botanica Brasilica 18: 781-792.

Schmitt, J. L. \& Windisch, P.G. 2010. Biodiversity and spatial distribution of epiphytic ferns on Alsophila setosa Kaulf. (Cyatheaceae) caudices in Rio Grande do Sul, Brazil. Brazilian Journal of Biology 70: 521-528.

Schuettpelz, E. \& Pryer, K.M. 2009. Evidence for Cenozoic radiation of ferns in an angiospermdominated canopy. Proceedings of the National Academy of Sciences of the United States of America 106: 11200-11205.

Schwartsburd, P.B. \& Labiak, P.H. 2007. Pteridófitas do Parque Estadual de Vila Velha, Paraná, Brasil. Hoehnea 34: 159-209.

Sehnem, A. 1977. As filicíneas do Sul do Brasil, sua distribuição geográfica, sua ecologia e suas rotas de migração. Pesquisas (Botânica) 31: 1-108.

Silva, A.T. 1984. Pteridófitas. In: Fidalgo, O. \& Bononi, V.L.R. Técnicas de coleta, preservação e herborização de material botânico. Insituto de Botânica, São Paulo. Pp. 31-34.

Smith, A.R.; Pryer, K.M.; Schuettpelz, E.; Korall, P.; Schneider, H. \& Wolf, P.G. 2006. A classification of extant ferns. Taxon 55: 705-731. 
Smith, A.R.; Pryer, K.M.; Schuettpelz, E.; Korall, P.; Schneider, H. \& Wolf, P.G. 2008. Fern classification. In: Ranker, T.A. \& Haufler, C.H. Biology and evolution of ferns and lycophytes. Cambridge University Press, New York. Pp. 417-467.

Testo, W. \& Sundue, M. 2014. Primary hemiepiphytism in Colysis ampla (Polypodiaceae) provides new insight into the evolution of growth habit in ferns. International Journal of Plant Sciences 175: 526-536.

Tryon, R.M. \& Tryon, A.F. 1982. Ferns and allied plants, with special reference to tropical America. Springer-Velarg, New York. 857p.
Veloso, H.P.; Filho, A.L.R.R. \& Lima, J.C.A. 1991. Classificação da vegetação brasileira, adaptada a um sistema universal. IBGE, Departamento de Recursos Naturais e Estudos Ambientais, Rio de Janeiro. 124p.

Watkins Jr., J.E.; Cardelús, C.; Colwell, R.K. \& Moran, R.C. 2006. Species richness and distribution of ferns along an elevational gradient in Costa Rica. American Journal Botany 93: 73-83.

Xavier, S.R.S. \& Barros, I.C.L. 2003. Pteridófitas ocorrentes em fragmentos de Floresta Serrana no estado de Pernambuco, Brasil. Rodriguésia 54: $13-21$. 